\title{
Luth pascalien et faiblesse humaine : des intrus dans la « raison des effets »?
}

Christian Meurillon

\section{(2) OpenEdition}

\section{Journals}

Édition électronique

URL : http://journals.openedition.org/ccibp/552

DOI : $10.4000 /$ ccibp. 552

ISSN : 2493-7460

Éditeur

Centre international Blaise Pascal

\section{Édition imprimée}

Date de publication : 19 octobre 1999

Pagination : 47-54

ISSN : 0249-6674

\section{Référence électronique}

Christian Meurillon, «Luth pascalien et faiblesse humaine : des intrus dans la « raison des effets »? », Courrier du Centre international Blaise Pascal [En ligne], 20 | 1999, mis en ligne le 06 janvier 2016, consulté le 01 mai 2019. URL : http://journals.openedition.org/ccibp/552 ; DOI : 10.4000/ccibp.552

Ce document a été généré automatiquement le 1 mai 2019.

Centre international Blaise Pascal 


\title{
Luth pascalien et faiblesse humaine : des intrus dans la « raison des effets »?
}

\author{
Christian Meurillon
}

1 Habituellement, la liasse des Pensées intitulée « Raison des effets » est envisagée selon le point de vue de la force. La force y est d'abord un objet d'étude pour l'apologiste : il montre que, guerrière d'origine, elle en vient cependant à bâtir, pour mieux asseoir sa domination, par une sorte de ruse objective de la charité, le «si bel ordre » (L106 - S1381) d'une société pacifiée en régnant à travers imagination et coutume ${ }^{2}$. La force est aussi, du côté du sujet connaissant, cette qualité d'esprit des « habiles » et a fortiori des « chrétiens parfaits» qui s'avèrent capables de saisir la coexistence surprenante de faits contradictoires en la pensant comme effet et de remonter jusqu'à la raison qui l'explique ${ }^{3}$.

2 À ces perspectives aussi fécondes que dorénavant bien connues, je me propose de substituer ici une approche par la "faiblesse", approche de biais mais non sans implications théologiques et épistémologiques. Elle permet en effet d'envisager la « Raison des effets » en négatif : la faiblesse est un objet dont celle-ci doit rendre compte comme elle le fait de la force; la faiblesse, comme la force, caractérise l'habile. L'unique fragment de cette liasse qui la mentionne explicitement (L96 - S130) retiendra spécialement notre attention.

3 Un examen préalable des trois grands dictionnaires de langue du XVII siècle laisse une impression accablante. Ces ouvrages ne voient en effet dans la «faiblesse » rien d'autre que la manque ou l'absence de force, d'abord physique puis, au sens figuré, morale. Ils ne suggèrent de plus que de rares synonymes : l'Académie propose « imbécile ", « débile », et "défectueux »; Furetière se contente d'« imbécile »; Richelet reste coi ${ }^{4}$. On peut passer sur le détail pour souligner deux traits généraux. D'une part, le sens figuré est attesté beaucoup plus dans le champ de la morale que dans celui de l'intelligence, même quand il est question d'esprit en général. Dans ce dernier cas, un « esprit faible » est un esprit « qui se laisse aisément persuader, qui ne peut soutenir les adversités, qui se scandalise facilement» (Furetière), ou qui «reçoit facilement toute sorte d'impressions » 
(Académie). L'antonyme de «faiblesse» est alors «fermeté ». D’autre part, ces dictionnaires ne présentent absolument aucun emploi de «faible » ou de «faiblesse » en contexte théologique ou même vaguement religieux. On peut en conclure que la faiblesse ne relève pour eux que d'un imaginaire linguistique profane.

L'examen du Dictionnaire historique de la langue française d'A. Rey redouble notre accablement tout en fournissant une explication à la relative pauvreté des articles précédemment examinés : en français en effet, depuis l'origine, «faible» signifie sans cesse et uniquement "qui manque de force ${ }^{5}$, avec simplement un élargissement progressif du domaine physique au domaine moral puis au domaine intellectuel. La notice historique du Trésor de la langue Française à l'article «faible » confirme ces informations. Mais elle réserve une agréable surprise. Pascal y est crédité d'une initiative linguistique tellement heureuse qu'elle est passée dans l'usage et qu'elle semble appartenir au fond immémorial de notre phraséologie. Il est en effet le premier à avoir substantivé l'adjectif pris dans son sens abstrait : c'est à partir des Pensées et de son expression «l'imagination des faibles» (L44 - S78) qu'« un faible " peut être un homme «qui manque de vigueur intellectuelle ou morale ${ }^{\natural} »$. On doit voir là un indice que Pascal a retravaillé l'emploi de ce mot courant, indice qui suggère l'existence d'un idiolecte voire d'une pensée originale en matière de faiblesse.

5 Selon la même méthode d'approche progressive concentrique, une étude des emplois de «faiblesse » dans l'ensemble des Pensées permet de dégager plusieurs caractères significatifs.

6 Le premier caractère concerne la répartition des occurrences du terme. Autant « force » est un des termes vedettes de la liasse " Raison des effets », autant « faiblesse », qu'on n'y rencontre que deux fois (et encore dans le même bref fragment L96 - S130), apparait surtout dans les autres liasses ou dossiers, sans qu'on puisse percevoir de règle de dispersion. La liasse « Raison des effets » se singularise curieusement par sa pauvreté en la matière. Ensuite, jamais le terme «faiblesse » ne qualifie le cœur ou la volonté et n'a de sens strictement moral. Plusieurs fois il désigne le manque de force d'un corps. Le sens intellectuel prédomine - tel était d'ailleurs significativement le sens de l'emploi substantivé de l'adjectif par Pascal - ; on n'est pas surpris de le rencontrer à propos des pyrrhoniens et des indifférents ${ }^{7}$.

7 Enfin, la faiblesse caractérise l'homme en tant qu'objet d'étude pour l'apologiste. Il s'agit d'abord de la faiblesse de la créature en tant que telle: son corps n'est qu'un point englouti par la nature, avec laquelle il n'a pas de proportion ${ }^{8}$. La dignité et la supériorité de l'homme résident dans sa pensée, qui est conscience de cette faiblesse ${ }^{9}$. La faiblesse du pécheur, qui est effet de la Chute, s'ajoute à la précédente et en reproduit la structure.

Sans ces divines connaissances qu'ont pu faire les hommes sinon ou s'élever dans le sentiment intérieur qui leur reste de leur grandeur passée, ou s'abattre dans la vue de leur faiblesse présente ? (L208 - S240 ${ }^{10}$.

En dehors de cet exemple, Pascal préfère cependant employer d'autres termes pour former des couples de contraires avec "grandeur ": "misère" (le plus souvent), "petitesse» (rarement) et «bassesse ${ }^{11}$ ». La faiblesse proprement dite semble spécialement associée à la condition de l'homme en ce qu'elle est «faible et mortelle et si misérable » (L136 - S168), tant il est vrai que la mort découle du péché. En cette rencontre avec la faiblesse, la mort est chaque fois colorée en « agonie » :

Est-ce courage à un homme mourant d'aller, dans la faiblesse et dans l'agonie,

affronter un Dieu tout-puissant et éternel ? (L432 - S662) 
Pourquoi le font-ils si faible dans son agonie? (L316 - S347) fait homme au point de mourir, c'est un imaginaire pathétique qui s'impose. Dans le même ordre d'idée, on peut remarquer que le sens de l'étymon "flebilis », non conservé par «faible », resurgit discrètement dans l'expression « condition déplorable » (L208 S240).

10 En première conclusion, « faiblesse » désigne surtout un état général de l'homme, objectif et non ressenti (contrairement à « misère »). En ce sens, ce terme ne s'oppose nullement à «force »: «force de l'homme » n'appartient pas à la phraséologie des Pensées, non plus que « les forts ». Il faut chercher ailleurs les antonymes de faible. Symétriquement, force entre en relation d'opposition avec de multiples termes autres que faiblesse (raison, opinion, imagination, fantaisie et grimace notamment ${ }^{12}$ ). On sait que Pascal affectionne la formation de tels couples de notions et fonde le dynamisme de nombreux fragments sur le jeu entre les deux pôles de ces antithèses. Or, dans cette fonction d'antonyme à fort, le banal et attendu « faible » est remplacé systématiquement par « juste » (L103 - S135 etc.). Mais le lecteur des Pensées peut-il encore percevoir le caractère proprement pascalien de cette dyade formée par «fort » et «juste», tellement celle-ci a été intégrée à notre culture et est devenue à son tour courante?

11 En seconde conclusion, «faiblesse » a un double usage. Remplaçable par «misère » et opposable à « grandeur », « faiblesse » s'applique à un aspect de la nature humaine, union indissociable des contraires misère et grandeur qu'étudient les liasses «Grandeur » et «Contrariétés » placées à la suite de « Raison des effets », et qu'explique ensuite la liasse «APR » par la voix de la Sagesse de Dieu. Mais, en même temps, «faiblesse » peut s'appliquer à la contrariété elle-même. Ce terme ne désigne pas alors un versant de l'état contradictoire de l'homme, ni une moitié de sa nature monstrueuse offerte comme une irritante énigme au philosophe et au moraliste, mais la totalité de la condition humaine ${ }^{13}$ telle que le chrétien la comprend. Chez Pascal - et nous découvrons ici l'idiolecte envisagé au départ par l'hypothèse -, « faiblesse » appartient au langage de l'apologiste qui subsume sous ce concept les caractères contraires de l'homme déchu, qui est à la fois misérable et grand de ne pas se satisfaire de cette misère. La faiblesse se trouve alors lourdement chargée d'un arrière-plan théologique. Dès les premières apparitions de ce terme, dans les liasses qui traitent des conceptions de l'homme élaborées selon les simples lumières naturelles, le lecteur bien informé saisit la "pensée de derrière » d'un auteur muni de la clef de l'énigme par la Révélation de cette chute qui abîme l'état de création de l'homme sans en effacer le souvenir.

12 La lexicologie historique avait déjà relevé dans les Pensées le premier emploi substantivé de «faible » au sens intellectuel et moral. L'analyse idéologique du lexique, quant à elle, fait apparaître un élargissement encore plus important : «faiblesse » appartient aussi au domaine de la théologie. Ce mot n'y désigne pas un caractère humain à examiner mais constitue d'emblée et à soi seul un jugement porté sur l'origine de l'état actuel de l'homme.

13 Si la faiblesse est la marque de l'homme comme objet de connaissance, on doit s'attendre à ce qu'elle affecte ses capacités comme sujet connaissant. On observe en effet le même glissement en matière d'épistémologie que de théologie. La force d'esprit ne s'oppose pas plus à la faiblesse d'esprit que la faiblesse ne s'oppose à la force. Comme la force dans la faiblesse, elle est, chez Pascal, englobée dans ce qui lui est ailleurs opposé. Qu'est-ce en 
effet qu'un « esprit fort » sinon un faible d'esprit? Il faudrait en effet être étrangement fort pour fonder l'athéisme sur des raisons convaincantes :

Athéisme marque de force d'esprit

Mais jusqu'à un certain degré seulement. (L157 - S189 $\left.9^{14}\right)$.

En fait, la raison, en se voulant forte, se discrédite en matière de vérité comme de souverain bien $^{15}$ : elle

n'est que faible si elle ne va jusqu'à reconnaître cela [qu'il y a une infinité de choses qui la surpassent]. (L188 - S220)

Et

rien n'accuse davantage une extrême faiblesse d'esprit que ne pas connaître quel est le malheur d'un homme sans Dieu. (L427 - S681)

Une faiblesse gnoséologique s'ajoute donc à la faiblesse ontologique dans l'attitude des pyrrhoniens :

la faiblesse de l'homme parait bien davantage en ceux qui ne la connaissent pas qu'en ceux qui la connaissent. (L34 - S68)

La véritable force consiste au contraire

à savoir douter où on il faut, assurer où on il faut, en se soumettant où on il faut.

Qui ne fait ainsi n'entend pas la force de la raison. L170 - S201)

Il ne faut pas voir en tout cela un renversement évangélique des fausses valeurs humaines, sur le modèle des Béatitudes, ni un effet de la pensée de la folie de la croix, en face de laquelle les sagesses humaines apparaissent pour ce qu'elles sont, des folies qui éloignent de la recherche de Dieu. Il s'agit plutôt d'une inclusion : si le fort est un faible, le faible n'en reste pas moins faible, même s'il l'est moins. Force et faiblesse ne sont que les deux faces d'une même faiblesse.

17 Lisons enfin la liasse "Raison des effets» dans la perspective théologique et épistémologique qui vient d'être esquissée.

18 La notion de faiblesse relève principalement de l'analyse politique qui montre qu'elle participe, autant que la force des forts, à la formation des sociétés ordonnées. Elle y joue le rôle de victime, comme le montre Pascal en imaginant l'origine de la société entre les hommes :

Il est sans doute qu'ils se battront jusqu'à ce que la plus forte partie opprime la plus faible et qu'enfin il y ait un parti dominant. (L828 - S668)

19 Cependant, non seulement elle bénéficie finalement de la paix imposée par la force, mais encore elle collabore, sans le savoir il est vrai, à la pérennisation de cet admirable «tableau de charité » (L118 - S150) qu'est l'ordre ainsi instauré. Un trait essentiel de la nature humaine concourt en effet à l'établissement de celui-ci : l'imagination. Elle est la continuation chez l'adulte des jeux de l'esprit enfantin :

le moyen que ce qui est si faible étant enfant soit bien fort étant plus âgé ? [...] Tout ce qui a été faible ne peut jamais être absolument fort. On a beau dire : Il est cru, il est changé ; il est aussi le même. (L779 - S643 $\left.{ }^{16}\right)$

L'enfant qui s'effrayait du « visage qu'il [avait] barbouillé » persiste chez l'adulte. Celui-ci «ne fait que changer de fantaisie» sans cesser d'être gouverné par l'imagination. Voilà donc paradoxalement le socle sur lequel se bâtit l'ordre arbitraire mais pacifique des sociétés. C'est particulièrement le cas des systèmes monarchiques, où la puissance des rois

a pour fondement la faiblesse. Et ce fondement est admirablement sûr, car il n'y a rien de plus sûr que le peuple sera faible. Ce qui est fondé sur la seule raison est bien mal fondé [...]. (L26-S60) 

caractère raisonnable de l'existence d'un maître, encore faut-il que leur raison n'aille pas jusqu'à exiger de choisir selon ses mérites qui sera le maître, car chacun dira qu'il est le plus apte. Mieux vaut donc que le peuple s'imagine que seule une naissance royale autorise à occuper le trône. Il agit sainement, car il maintient la paix, qui est ici-bas le plus grand bien, en évitant de donner l'occasion à la force de s'exercer dans sa brutalité. La Fronde a montré d'autre part que ceux qui se targuent de force d'esprit, par exemple en prétendant atteindre une véritable justice humaine ou revenir à une prétendue pureté originelle des lois, ne font dans la pratique que causer des guerres civiles parce que la justice n'est pas une qualité palpable indiscutable. Sur le plan de la théorie, ces demihabiles ne peuvent rendre compte de la fonction pacifiante de la coutume et de l'imagination, contrairement aux habiles, ni de l'origine de la coexistence en l'homme d'une avidité de vraie justice et d'une incapacité à l'atteindre, contrairement aux chrétiens parfaits. Heureuse et sage faiblesse d'esprit de ceux qui sont impressionnés par les signes de la force véridiques - parfums, brocatelle et laquais des riches ${ }^{17}-$, ou trompeurs - ornements masquant l'absence de force, de justice ou de savoir des magistrats et des médecins ${ }^{18}$-, et qui respectent et « honore[nt] les personnes de grande naissance » (L90 - S124) !

Le peuple, ce grand enfant, barbouille les lois d'une justice qu'il imagine, barbouille le visage des agents du roi d'une force qu'ils n'ont pas et celui du roi d'une force qui n'appartient qu'à ses troupes. Le barbouillage, aussi bénéfique que ridicule aux yeux de l'habile, voilà finalement l'activité politique du peuple que donne à voir l'ensemble de la liasse « Raison des effets ».

Il faut enfin examiner tout particulièrement le rapport entre faiblesse et beauté parce qu'il apparaît dans le seul fragment de la liasse qui présente une occurrence de «faiblesse » (L96 - S130), mais sans oublier que c'est toujours la même question qui est envisagée : l'effet de la faiblesse, qui est dans le reste de la liasse la paix continue, est dans ce fragment la beauté. Beauté et paix sont en effet à la fois des biens estimables à l'intérieur de l'ordre humain et de simples valeurs d'établissement artificielles au regard de ce que serait l'harmonie d'une véritable cité de Dieu.

Examinons la genèse et les enjeux de ce modeste fragment, écarté par les éditeurs de Port-Royal, publié tardivement (par Faugère, en 1844), ayant résisté à l'enquête paléographique menée par P. Ernst ${ }^{19}$ et n'ayant jamais fait l'objet, à ma connaissance, d'un examen spécifique attentif.

Recourons d'abord à la source que propose Brunschvicg dans sa grande édition des Pensées $^{20}$, à savoir l'essai de Montaigne intitulé «Des vaines subtilités ». Dès le premier paragraphe de cet essai, Montaigne dégage en effet d'exemples divers, notamment celui de poèmes à forme imposée, un enseignement général dont Pascal pouvait directement tirer profit :

C'est un témoignage merveilleux de la faiblesse de notre jugement, qu'il recommande les choses par la rareté ou nouvelleté, ou encore par la difficulté, si la bonté et utilité n'y sont jointes ${ }^{21}$.

L'intérêt de cette remarque cependant, qui ne s'impose pas indubitablement comme source nécessaire et suffisante, réside essentiellement dans le contexte que lui offre l'essai qui l'accueille. En effet, Montaigne ajoute immédiatement :

Nous venons présentement de nous jouer chez moi à qui pourrait trouver plus de choses qui se tiennent par les deux bouts extrêmes : comme Sire, c'est un titre qui

Courrier du Centre international Blaise Pascal, 20 | 1999 
se donne à la plus élevée personne de notre État, qui est le Roi, et se donne aussi au vulgaire, comme aux marchands, et ne touche point ceux d'entre deux. Les femmes de qualité, on les nomme Dames; les moyennes, Demoiselles; et Dames encore, celles de la plus basse marche. On voit que, dans ce contexte à la fois ludique et politique, se précise une idée de
tripartition: dans un domaine hiérarchisé donné, il est constaté que les premier et
troisième degrés sont similaires et que le degré moyen constitue un cas à part. du même type. Montaigne s'attache tout particulièrement à noter les cas où les deux extrêmes se rejoignent en produisant des effets identiques (par exemple, «l'extrême chaleur et l'extrême froideur cuisent et rôtissent ») et à montrer les défauts de la position intermédiaire. Il distingue ainsi trois « étages » de chrétiens : " esprits simples », esprits de « moyenne vigueur » et " grands esprits ${ }^{22}$ ». De même, la poésie comporte trois degrés : autant la "poésie populaire » et la "poésie parfaite selon l'art » sont comparables en grâce et beauté, autant la " poésie médiocre " ne mérite que dédain. De même, "paysans simples » et " philosophes » sont les uns et les autres "honnêtes gens », tandis que les «métis [...] (le cul entre deux selles) [...] troublent le monde ». Ces « métis », on le sait, sont la source directe de la notion de demi-habile si caractéristique de la pensée pascalienne. Mais ce qui apparaît le plus remarquable est la réunion dans un même contexte des traits essentiels de la gradation des effets : échelonnement, tripartition et exclusion du milieu, lequel additionne les défauts des deux extrêmes mais non leurs qualités ${ }^{23}$. Ces traits cependant, s'ils structurent sa réflexion, ne sont pas pensés comme tels par Montaigne et ne seront donc pas systématiquement réemployés. Il reviendra à Pascal de faire l'un et l'autre.

On comprend alors mieux la présence du fragment L96 - S130 dans la liasse « Raison des effets ». Loin de constituer un exemple quelconque, voire marginal par rapport aux effets d'importance majeure relevés en matière politique, ce fragment est au contraire, oserai-je affirmer, le point focal de toute la liasse, la trace discrète en son sein de la genèse de la " raison des effets", et particulièrement des notions de "gradation » et de "demihabile ", à partir de la lecture d'un essai lui-même discret de Montaigne.

Ce fragment n'est pas pour autant d'interprétation facile. Pour preuve, la multiplicité des versions qu'en proposent les différents éditeurs des Pensées (voir en annexe), faute de pouvoir arriver à un texte à la fois incontestable et satisfaisant pour le sens à partir du manuscrit. Si le déchiffrement mot à mot est aisé, la syntaxe reste obscure, au point qu'on est tenté de voir dans la phrase finale de Pascal la collusion de ses deux états successifs dans le temps. Essayons d'établir et d'interpréter chacune de ces strates d'écriture.

31 La question est de savoir ce qui est un mal : est-ce « ne point jouer du luth » ou bien est-ce « savoir bien en jouer »? Je me place dans l'hypothèse où Pascal a ajouté « ne... que » dans sa seconde rédaction, selon la lecture proposée par Tourneur dans son édition paléographique des Pensées $^{24}$.

Soit le premier jet hypothétique :

*La faiblesse de l'homme fait la cause de tant de beautés qu'on établit, comme de ne point jouer du luth est un mal à cause de notre faiblesse.

Une affirmation générale est suivie et éclairée non d'un exemple particularisant mais d'un exemple a contrario. La faiblesse est en effet à la fois la cause de beautés qui ne sont pas naturelles mais seulement d'établissement (comme c'est le cas, selon l'exemple donné dans la suite de la phrase, de la pratique du luth, appréciée la société aristocratique), et la 
cause du mal correspondant, qui est également d'établissement (il faut en effet savoir jouer du luth, selon les mêmes attentes d'une société qui, sur la base de ses valeurs purement humaines, blâme l'inaptitude au luth). Cette version est parfaitement cohérente : il existe des beautés d'établissement qu'il convient sans nul doute de saluer extérieurement; une condamnation sociale frappe ceux qui ne les respectent pas, par exemple en ne jouant pas du luth; si l'homme n'était pas corrompu, il n'aurait pas à devoir s'adonner à ce divertissement.

Soit le second jet hypothétique :

*La faiblesse de l'homme est la cause de tant de beautés qu'on établit comme de savoir bien jouer du luth n'est un mal qu'à cause de notre faiblesse.

Pascal fait maintenant apparaître la présence d'un effet surprenant, à savoir la coexistence de deux appréciations différentes causées par la même faiblesse. Il voit le parti qu'il peut en tirer: un "renversement du pour au contre», ce qui justifiera le classement de ce fragment dans la liasse «Raison des effets ». Mais il ne déploie pas ce renversement comme il le fait au fragment L93 - S127; il se contente d'en noter le principe de manière elliptique. Il relie directement l'exemple du luth à la première assertion : il doit donc supprimer la négation. Mais la seconde partie de la phrase est maintenant mal raccordée à la première. Les éditeurs adoptent différentes solutions. Lafuma et M. Le Guern proposent le texte tel quel ${ }^{25}$. D'autres tentent de sauver la situation : Brunschvicg et Ph. Sellier coupent la phrase, le premier ajoutant « ce » sans le signaler $^{26}$, le second restituant entre crochets l'élément marquant; E. Martineau additionne les deux états du texte ${ }^{27}$.

Mais pourquoi ne pas tirer profit de la superposition partielle de deux phrases - un élément étant mis en facteur commun -et ne pas voir dans ce fragment l'affirmation qu'une même chose, jouer du luth, peut être justiciable de deux jugements différents et qu'une même cause, la faiblesse, peut produire à la fois un jugement valorisant et un jugement dépréciatif (en l'occurrence, sur l'aptitude à jouer du luth), jugements opposés mais justifiables à des niveaux différents?

Dans cette perspective, suivons le modèle de la gradation de la raison des effets et déployons le fragment en question comme un papier plié japonais jeté dans une tasse de thé.

Jouer du luth ${ }^{28}$ est une beauté réelle pour le monde, qui est sensible au plaisir des sens et à la distinction sociale : la musique rend heureux les gens des salons en montrant qu'ils ont du loisir, de la fortune et du talent.

Un demi-habile n'aurait pas de mal à montrer que c'est une simple beauté conventionnelle et se moquerait des jugements mondains. Une autre société privilégierait en effet d'autres activités artistiques ou ludiques :

La force est la reine du monde, et non pas l'opinion [...]. C'est la force qui fait l'opinion. La mollesse est belle, selon notre opinion. Pourquoi? Parce que qui voudra danser sur la corde sera seul. Et je ferai une cabale plus forte de gens qui diront que ce n'est pas beau. (L554 - S463)

L'aristocratie médiévale a imposé son choix du troubadour et de ses molles chansons amoureuses; elle aurait pu tout aussi bien préférer le danseur de corde. Une fronde pourrait imposer de force ses choix. Et les Suisses, si épris de roture ${ }^{29}$, que choisiraientils ? Les clarines, la trompe de montagne...? 
41 L'habile joue le jeu de l'honnêteté, même dans le salon de Célimène, parce qu'il en apprécie, comme Miton, la commodité sociale ${ }^{30}$. Il sait que le luth contribue à assurer l'harmonie des relations et à pacifier la société.

Pour le dévot, au contraire, jouer du luth est un mal réel, résultat de la faiblesse humaine, et condamnable au même titre que toutes ces distractions mondaines qui satisfont la vanité de l'amour-propre et détournent l'âme de la recherche de Dieu. C'est en ce sens que Pascal utilise cette activité comme exemple de « divertissement » :

à quoi pense le monde ? Jamais à cela! Mais à danser, à jouer du luth, à chanter, à

faire des vers, à courir la bague, etc., à se battre, à se faire roi, sans penser à ce que

c'est qu'être roi, et qu'être homme. (L620 - S513)

Un dévot honnête homme au contraire, comme saint François de Sales dans son Introduction à la Vie dévote, sait, comme les "chrétiens parfaits » de la gradation, que l'esprit et le corps ne peuvent être sans cesse tendus vers Dieu :

Prendre l'air, se promener, s'entretenir de devis joyeux et amiables, sonner du luth ou autre instrument, chanter en musique, aller à la chasse, ce sont récréations si honnêtes que pour en bien user il n'est besoin que de la commune prudence ${ }^{31}$ [...].

Pascal me semble cependant développer un point de vue plus radical. Il faut donner à la restriction «ne...que » - qu'elle soit du premier ou du second jet - toute sa valeur : jouer du luth n'est un mal que parce que l'homme est faible; sinon, ce serait franchement un bien. Non parce qu'une compétence serait en soit un bien : encore faut-il qu'elle concerne une activité qui soit elle-même bonne. C'est ce dernier cas qui est ici suggéré. On sait d'ailleurs que les Pascal tenaient la musique en haute estime ${ }^{32}$. Ici, Blaise Pascal semble regretter que la faiblesse des hommes barbouille d'une fausse beauté d'établissement la beauté naturelle de la musique et, plus profondément, que la corruption de l'homme rende dangereux un passe-temps qui, autrement, serait profitable pour son salut. Il ne doit pas manquer d'admirer que la faiblesse humaine réussisse pour de mauvaises raisons de vanité à favoriser la beauté musicale. Le chrétien parfait songe sans doute au concert des anges musiciens ou aux «harpes » de l'Apocalypse ${ }^{33}$, dont jouent les sept anges ainsi que les vieillards qui entourent l'Agneau. Dans l'état de gloire, la musique tourne vers Dieu.

Le luth amoureux et mondain apparaît alors dans toute sa puissance figurative de signe, prêt de surcroît à se transfigurer comme chose en instrument céleste. Entre les deux, il faut postuler l'aptitude spéciale du joueur de luth à la conversion.

\section{ANNEXES}

\section{Choix d'éditions du fragment L96 - S130}

\section{- Brunschvicg 329 :}

« Raison des effets. - La faiblesse de l'homme est la cause de tant de beautés qu'on établit, comme de savoir bien jouer du luth. 
Ce n'est un mal qu'à cause de notre faiblesse. »

- Tourneur :

«[La foiblesse de]

Raison des effets

La foiblesse de 1 homme [fait] est la Cause de tant de beautez qu on establit Comme de [ne point] scauoir bien Jouer du luth n'est Vn mal qu'a Cause de nostre foiblesse. »

\section{- Lafuma 96 et Le Guern 89 :}

« Raison des effets.

La faiblesse de l'homme est la cause de tant de beautés qu'on établit, comme de savoir bien jouer du luth n'est un mal qu'à cause de notre faiblesse. »

- Sellier 130 :

« Raison des effets.

La faiblesse de l'homme est la cause de tant de beautés qu'on établit, comme de savoir bien jouer du luth. [Ne point jouer du luth] n'est un mal qu'à cause de notre faiblesse. »

- Martineau XV :

«La faiblesse de l'homme est la cause de tant de beautés qu'on établit, comme de « ne point » savoir bien jouer du luth n'est un mal qu'à cause de notre faiblesse. »

\section{Note de la rédaction}

La communication de Christian Meurillon a donné lieu à un échange de lettres qui nous a paru tout à fait dans l'esprit pascalien. Avec l'accord des deux intéressés, nous publions ciaprès la lettre par laquelle Jean-Claude Pariente communiquait ses réflexions, qui reflète bien l'ambiance amicale de cette journée " Raison des effets ».

Le 13 juin 1997

Monsieur et cher collègue,

Votre communication de samedi dernier m'a beaucoup intéressé et m'a vivement stimulé. J'ai été très sensible à la façon excitante dont vous avez proposé plusieurs hypothèses interprétatives pénétrantes à propos du fragment $\mathrm{Br}$. 329. J'étais pour ma part jusqu'à votre exposé complètement privé de réactions devant ce fragment qui ne m'inspirait que de la perplexité.

Vos analyses m'ont paru convaincantes dans l'hypothèse de travail que vous avez adopté, selon laquelle ne...que serait une addition contemporaine de la substitution de savoir bien à ne point. Mais que dire si ne...que n'est pas une addition?

En y réfléchissant dans la semaine, je me suis dit qu'un des critères auxquels devait satisfaire une interprétation était le suivant : elle doit proposer un moyen de comprendre que Pascal ait pu subsister savoir bien à ne point sans modifier le reste de la phrase ; en d'autres termes, il faut proposer une interprétation qui permette de comprendre que n'est un mal qu'à cause de notre faiblesse puisse être attribut de savoir bien jouer du luth autant que de ne point jouer du luth. Si cette condition n'est pas respectée, il devient, me semble-t-il, inacceptable que Pascal ait pu prédiquer la même chose de deux états de choses incompatibles, celui dans lequel on sait bien jouer du luth, et celui dans lequel on n'en joue pas. 
Voici la suggestion que je vous soumets pour répondre à cette condition - quand on admet que le ne... que n'a pas été ajouté dans la seconde rédaction.

Dans la première partie du fragment (jusqu'à « qu'on établit »), Pascal attribue à notre faiblesse l'ignorance où nous sommes d'après $\mathrm{Br} .33$ du modèle naturel qu'il faudrait imiter; d'où l'idée que les beautés sont établies (les reines de village de 33).

Cela étant, il donne d'abord à la deuxième partie la formulation : comme de ne point jouer du luth n'est pas un mal qu'à cause de notre faiblesse. Deux points sont à expliquer, a) le contenu de cette affirmation, et b) les raisons qui amènent Pascal à penser qu'il y a dans cette affirmation quelque chose de parallèle (comme) à l'établissement des beautés.

Sur a), je crois que les choses sont simples. Pascal veut dire : si nous n'étions pas aussi faibles, ou vains, nous ne considérerions pas comme un mal le fait de ne pas jouer du luth.

Sur b), je propose ce qui suit : nous sommes en présence de la même situation que pour les beautés. De même que nous établissons ce qui est beau, nous établissons ce qui est mal, et, pour illustrer, il prend l'exemple d'une inaptitude qui est en réalité anodine, mais qui n'en est pas moins de quelque conséquence dans un monde régi par la vanité. Le parallèle porte sur l'établissement : ne point jouer du luth n'est pas un mal authentique, c'est un mal par établissement, comme les reines de village sont des beautés d'établissement.

Mais il s'avise que la comparaison est boiteuse. En effet, dans la première partie, ce qui est établi est porteur d'une valence positive, c'est une beauté. Dans la seconde partie, l'établissement porte sur du négatif (ne point jouer). Or on pourrait facilement rétorquer : inutile d'invoquer la faiblesse pour expliquer que ce soit un mal de ne point jouer du luth ; une ignorance, ou une inaptitude, est par elle-même un mal. Si, comme on a semblé l'accorder samedi dernier, un effet doit être surprenant par quelque côté, que ce soit un mal de ne point jouer du luth n'a pas le statut d'effet. Pascal cherche alors au plus près de l'exemple pris (jouer du luth) ce qui pourrait être constitué en mal dû à notre faiblesse tout en étant positif dans sa nature. Et il remplace ne point par savoir bien : il obtient ainsi un effet à expliquer, par l'affirmation que savoir bien faire quelque chose soit un mal.

Il faut, bien entendu, répondre pour la seconde rédaction aux mêmes questions a) et b) que pour la première.

Sur a), on peut proposer ceci : savoir bien jouer du luth est en soi une aptitude positive (une « beauté » peut-être) ; si elle devient un mal, ce n'est qu'à cause de notre faiblesse, en ce sens que seule notre faiblesse explique que nous greffions sur cette aptitude des comportements de vanité et qu'elle devienne ainsi une source ou une occasion de divertissement.

Sur b), je suggère que le parallèle entre les deux parties s'est déplacé quand on passe de la première à la seconde rédaction. Tout à l'heure il portait sur l'établissement. Maintenant il porte plutôt sur la causalité de la faiblesse : de même qu'elle est 
responsable de l'établissement des beautés, elle est responsable, et même seule responsable, du mal qui peut résider dans une aptitude positive.

Si ces considérations ne sont pas aberrantes, elles peuvent satisfaire, me semble-t-il, à la condition que je posais plus haut. Mes hypothèses sont moins riches que les vôtres, à coup sûr. Mais je ne les propose que pour résoudre le problème que pose ce fragment quand on admet que ne...que figurait dans la première rédaction. Cela explique en grande partie notre divergence. D'une certaine façon, nous nous complétons!

Je reste en tout cas votre débiteur car je n'osais plus toucher à ce fragment avant de vous avoir écouté.

Croyez, je vous prie, à l'expression de mes sentiments très cordiaux.

Jean-Claude Pariente

\section{NOTES}

1. Les références des Pensées sont données après chaque citation ou mention, successivement dans l'édition de L. Lafuma (Paris, Éd. du Luxembourg, 1952, 3 vol. ) et dans celle de Ph. Sellier (Paris, Bordas, « Classiques Garnier », 1991), dont je suis le texte.

2. Voir G. Ferreyrolles, Les Reines du monde. L'imagination et la coutume chez Pascal, Paris, Champion, «Lumière classique », 1995.

3. Voir L. Thirouin, « Raison des effets, essai d'explication d'un concept pascalien », XVII ${ }^{e}$ siècle, $\mathrm{n}$ -134, 1982, pp. 31-50.

4. Les Pensées, quant à elles, présentent trois occurrences de « imbécile » et aucune de « débile ».

5. Dictionnaire étymologique de la langue française, sous la direction d'Alain Rey, Paris, Dictionnaire Le Robert, 1992, T. 1, p. 774. Première attestation de «faible » au sens physique en 1080 ; au sens moral, en 1180.

6. Trésor de la langue Française, Paris, Éd. du Centre National de la Recherche Scientifique, T. 8, 1980, p. 587.

7. L'apologiste prête à l'indifférent des mots lourdement chargés de sa pensée de derrière : à la proclamation du second, "voilà mon état, plein de faiblesse et d'incertitude », répond l'accusation d' " extrême faiblesse d'esprit » de la part du premier (L427 - S681).

8. Voir L199 - S230 : " (Voilà une partie des causes qui rendent l'homme si imbécile à connaître la nature. [...]) / Enfin, pour consommer la preuve de notre faiblesse, je finirai par ces deux considérations [...]. »

9. Voir évidemment L200 - S231 : «L'homme n'est qu'un roseau, le plus faible de la nature, mais c'est un roseau pensant. »

10. On peut citer également les deux séquences suivantes : ambition, concupiscence, misère et injustice (L595 - S491) ; « homme plein de faiblesse, de misère, de concupiscence, d'orgueil et d'ambition » (L931 - S759).

11. Grandeur / misère: L122 - S155 etc.; grandeur / petitesse: L215 - S248, L692 - S571; grandeur / bassesse : L398 - S17, L119 - S151, L121 - S153, L357 - S389. « Bassesse » présente le sens le plus riche puisqu'il est explicitement relié à l'abaissement de l'homme déchu, conçu comme modification d'une situation initiale.

12. Voir L711 - S589 («Force /. Pourquoi suit-on la pluralité ? Est-ce à cause qu'ils ont plus de raison? Non, mais plus de force.»); L554 - S463 (« La force est la reine du monde, et non pas 
l'opinion. ») ; L828 - S668 (force / imagination) ; L767 - S632 (force / fantaisie) ; L797 - S650 (force / grimace).

13. L'index de l'édition Sellier le suggère : «Faiblesse " y renvoie à " Homme » (op. cit., p. 642).

14. Voir aussi L150 - S183 : «Les impies qui font profession de suivre la raison doivent être étrangement forts en raison. »

15. Voir L28 - S62 : «Faiblesse. / [...] Nous sommes incapables de vrai et de bien. »

16. Voir aussi L. 136 - S. 168.

17. Voir L. 89 - S 123 et L. 95 - S. 129.

18. Voir L. 87 - S. 121.

19. Le papier portant cet autographe n'a pu être identifié par Pol Ernst (Les Pensées de Pascal. Géologie et stratigraphie, Paris - Oxford, Universitas - Voltaire Foundation, 1996, p. 293) ni donc situé dans la stratigraphie qu'il propose.

20. Pensées, Paris, Hachette, «Les Grands Écrivains de la France », 1904, T. II, p. 248, note 6. Cette indication de source est reprise par B. Croquette (Pascal et Montaigne, Genève Droz, 1974) et E. Martineau (Blaise Pascal, Discours sur la religion et sur quelques autres sujets, restitués et publiés par E. Martineau, Paris, Fayard / Armand Colin, 1992). L. Lafuma et Ph. Sellier ne proposent pas de source.

21. Essais, I, 54, éd. Villey-Saulnier, Paris, P.U.F., 1965, p. 311 (orthographe modernisée).

22. Ibid., pp. 312-313.

23. Sur cette question, voir mon article «Les combinaisons pascaliennes ou les avatars de la pensée ternaire ", Kyoto-Genève, Équinoxe, n 6, été 1990, pp. 49-68.

24. Pensées de Blaise Pascal, Pais, Vrin, 1942, p. 191.

25. M. Le Guern prend cependant parti en note ; «L'interprétation de ce fragment est difficile. Il faudrait peut-être comprendre : «tant de beautés qu'on établit comme de savoir bien jouer du luth ». Pascal aurait alors omis par inadvertance de rayer ce qui suit lorsqu'il a corrigé son texte primitif «ne point jouer du luth» en «savoir bien jouer du luth» (Pensées, Paris, Gallimard, «Folio », 1977, T. I, pp. 271-272, note 1 du fragment 89).

26. Brunschvicg précise en note : "Pascal avait d'abord écrit ne point savoir : il a ensuite corrigé pour faire correspondre la seconde partie de sa phrase à la première : c'est une beauté de savoir bien jouer du luth. Mais la seconde phrase ne s'explique que par cette première rédaction : ce qui est un mal, c'est de ne point savoir jouer du luth » (op. cit., p. 248, note 5).

27. E. Martineau commente ainsi la suppression de la négation par Pascal: "Correction « moralisante », avec changement de point de vue ? Maladresse, plutôt.» (op. cit., p. 252)

28. On connaît les connotations lyriques et amoureuses du luth. Les dictionnaires restent cependant muets sur ce point. Furetière consacre au luth un long article, mais entièrement technique, musicologique et étymologique, à une notation ironique près : « On est plus longtemps à accorder un luth, qu'à en jouer. " L'Académie propose une simple définition qu'elle fait suivre de multiples locutions qui lui permettent d'énumérer les parties du luth.

29. Voir L. 50 - S. 83 et L. 828 - S. 668.

30. Voir L597 - S494.

31. Introduction à la Vie dévote, III, chap. XXXI (Des passetemps et récréations, et premièrement des loisibles et louables), éd. A. Ravier, Paris, Galimard, «Bibliothèque de la Pléiade », 1969, p. 220.

32. Voir J. Mesnard, «Pascal et la musique », dans Pascal. Textes du tricentenaire, Paris, Fayard, 1963, pp. 195-205.

33. Apocalypse de Jean (V, 8 ; XIV , 2 ; XV, 2), selon la traduction de la Bible de Sacy (éd. Ph. Sellier, Paris, Robert Laffont, «Bouquins ", 1990, pp. 1604, 1611 et 1612). On peut songer aussi à la mise en scène burlesque "d'un chant d'église» par Scarron dans son Roman comique lorsqu'il développe à plaisir l'épisode de la « dévote sérénade » donnée sous les fenêtres d'une hôtellerie par « deux méchantes voix » qui chantent, accompagnées des orgues, «Allons, de nos voix, et de nos 
luths d'ivoire, ravir les esprits» (Le Roman comique, éd. Magne, Paris, Garnier Frères, "Classiques Garnier », 1973, p. 103).

INDEX

Mots-clés : Pascal, raison des effets, Pensées, faiblesse, musique

Keywords : Pascal, reason, Pensées, effect, weakness, music

\section{AUTEUR}

\section{CHRISTIAN MEURILLON}

Maître de conférences

Université Lille III 